\title{
More efficient reversal of dabigatran inhibition of coagulation by activated prothrombin complex concentrate or recombinant factor VIIa than by four-factor prothrombin complex concentrate
}

Tomas Lindahl, Maria Wallstedt, Kerstin Gustafsson, Egon Persson and Andreas Hillarp

\section{Linköping University Post Print}

\section{Tweet}

N.B.: When citing this work, cite the original article.

Original Publication:

Tomas Lindahl, Maria Wallstedt, Kerstin Gustafsson, Egon Persson and Andreas Hillarp, More efficient reversal of dabigatran inhibition of coagulation by activated prothrombin complex concentrate or recombinant factor VIIa than by four-factor prothrombin complex concentrate, 2015, Thrombosis Research, (135), 3, 544-547.

http://dx.doi.org/10.1016/j.thromres.2014.12.019

Copyright: Elsevier

http://www.elsevier.com/

Postprint available at: Linköping University Electronic Press

http://urn.kb.se/resolve?urn=urn:nbn:se:liu:diva-115816 
More efficient reversal of dabigatran inhibition of coagulation by activated prothrombin complex concentrate or recombinant factor VIIa than by four-factor prothrombin complex concentrate

Tomas L. Lindahl ${ }^{1,2}$, Maria Wallstedt ${ }^{1}$, Kerstin M. Gustafsson ${ }^{2}$, Egon Persson $^{3}$ and Andreas Hillarp ${ }^{4}$.

1) Dept. of Clinical and Experimental Medicine, Linköping University, Linköping, Sweden

2) Dept. of Clinical Chemistry and Dept. of Clinical and Experimental Medicine, Linköping University, Linköping, Sweden

3) Novo Nordisk A/S, Novo Nordisk Park, Måløv, Denmark

4) Dept. of Clinical Chemistry, Halmstad Hospital, Halmstad, Sweden

Corresponding author:

Tomas L. Lindahl

Phone; +46101033227

Fax; +46101033240

E-mail tomas.lindahl@liu.se 


\section{Introduction}

In the future, an increasing numbers of patients will be on antithrombotic treatment with novel oral anticoagulants, the first in clinical use being the direct oral thrombin inhibitor dabigatran $\left(\operatorname{Pradaxa}^{\circledR}\right)$ for patients with non-valvular atrial fibrillation. A small percentage of the patients on dabigatran will experience serious bleeding or be in need of urgent surgery. In those instances, the clinicians need guidance how to treat the patient in order to arrest or prevent bleeding. In contrast to warfarin, none of the non-vitamin $\mathrm{K}$ antagonist oral anticoagulants (NOAC) have a specific antidote approved yet. Nevertheless, in a recent analysis of 5 phase III clinical trials, patients who experienced major bleeding with dabigatran required more red blood cells but received less plasma, experienced a shorter stay in the intensive care unit and had a trend towards lower mortality $(\mathrm{p}=0.051)$ compared with those who had major bleeding with warfarin [1]. An antibody directed towards dabigatran developed by BoehringerIngelheim has shown very promising results in vitro and in a rat model [2]. A phase III study has started recruiting patients (NCT02104947) but approval for use in humans will take years. Hemodialysis is effective in removing dabigatran, but the method is time-consuming and may lead to bleedings at insertion sites. In vitro studies, animal models and case reports on the use of recombinant factor VIIa (rVIIa), prothrombin complex concentrates (PCC) and activated prothrombin complex concentrates (APCC), reviewed by Majeed and Schulman recently [3], have reported both promising and disappointing results. Due to the clinical need there are recommendations or proposals on the use of PCC, APCC or rFVIIa in severe bleeding if other measures are insufficient, despite scarce supporting evidence (see national Swedish recommendations http://ssth.se/documents/vp/vpeng120213.pdf and references [3-10]). The aim of our study was to 1) provide the medical community with data on in vitro effects by different hemostatic agents to support decision-making on reversal of 2) inspire further testing in animal models and clinical studies.

Whole blood was spiked with the active substance dabigatran at expected peak concentrations and the effects of drugs potentially improving coagulation were tested. We utilized a viscoelastometric clotting assay using the instrument $\operatorname{ReoRox}^{\mathrm{TM}}$ and measured clot initiation and propagation velocity from a tissue factor covered surface using the instrument Thrombodynamics Analyzer ${ }^{\mathrm{TM}}$. The effects of PCC, APCC and rFVIIa were tested.

\section{Materials and Methods}

\section{Blood samples and spiking with dabigatran}


The analyses involved samples from up to twenty adult healthy volunteers (numbers in figure legends), both men and women were included. The blood samples were anonymized at time of blood collection. The procedure was approved by the local ethical committee, although no specific approval for this procedure of blood collection is required. Blood was collected in evacuated plastic tubes containing 1:10 volume of $0.109 \mathrm{~mol} / \mathrm{L}$ tri-sodium citrate as anticoagulant. Preparation of platelet poor plasma (PPP) and platelet-rich plasma (PRP) was performed by standard centrifugation techniques. Citrated blood was spiked with dabigatran dissolved in DMSO at $20 \mu \mathrm{g} / \mathrm{mL}$, resulting in a final DMSO concentration of $1 \%(\mathrm{v} / \mathrm{v})$ in all samples. One dabigatran concentration was used, $200 \mu \mathrm{g} / \mathrm{L}$, and a control sample without dabigatran was processed identically. The reversing drug was added in smallest possible volume and an equal volume of $0.9 \% \mathrm{NaCl}$ was added to control tubes in order to compensate for the dilutional effect of the reversing drug. Spiking was checked by analysis of plasma using the calibrated Hemoclot Direct Thrombin inhibitor assay (Hyphen, NeuvilleSur-Oise, France) utilizing an ACL Top instrument (IL, Milan Italy).

\section{Reversal drugs tested}

The chosen concentrations are based on typical clinical dosage for reversal of warfarin (PCC) or treatment of hemophilia with inhibitors (rFVIIa, APCC). All drugs were reconstituted according to the recommendations of the manufacturer.

For recombinant activated factor VII (NovoSeven’ Novo Nordisk, Bagsværd, Denmark), standard treatment of $90 \mu \mathrm{g} / \mathrm{kg}$ was chosen, and in some experiments the concentration was tripled corresponding to high-dose treatment. This represents final concentrations in blood of $2.1 \mu \mathrm{g} / \mathrm{mL}$ and $6.3 \mu \mathrm{g} / \mathrm{mL}$, respectively.

Activated prothrombin complex concentrate, FEIBA ${ }^{\circledR}$ (Baxter AG, Vienna, Austria), was used at a final concentration of $1.8 \mathrm{U} / \mathrm{mL}$ of blood.

Prothrombin complex concentrate (PCC), Cofact $^{\circledR}$ (Sanguin, Amsterdam, the Netherlands) was used. Based on the treatment regimen of $40 \mathrm{U} / \mathrm{kg}$ we added $0.56 \mathrm{U} / \mathrm{mL}$ blood, i.e. $22.4 \mu \mathrm{L}$ per mL blood.

\section{Measurements of visco-elastic parameters}


Viscoelastometric measurements were performed on whole blood using free oscillation rheometry (FOR, determined by two four-channel ReoRox $2^{\mathrm{TM}}$ instrument from Medirox $\mathrm{AB}$, Nyköping, Sweden). We used disposable polypropylene sample cups and software (ReoRox ${ }^{\circledR} 4$ v. 2.00 and ReoRox ${ }^{\circledR} 4$ Viewer v. 2.11 k) from the manufacturer. In this rheometer, oscillation is initiated by a forced turn of the sample cup every 2.5 seconds. After a brief hold time the sample cup is released allowing rotational oscillation with very low friction around the longitudinal axis. The frequency and damping of the oscillation are measured as a function of time. Fibrin formation increases the viscosity of the sample, which leads to a decrease in the frequency and an increase in the dampening of the oscillation. The clotting time measured correlates extremely well with the manual reference method of visual clot detection [11]. Human recombinant thromboplastin (Dade Innovin ${ }^{\mathrm{TM}}$, from Siemens Healthcare Diagnostics Products GmbH, Marburg, Germany) was used as activator, final concentration of tissue factor $1.4 \mathrm{pmol} / \mathrm{L}$. The assay takes $0.7 \mathrm{~mL}$ blood per test, which was recalcified with $20 \mu \mathrm{L}$ of $500 \mathrm{mmol} / \mathrm{L} \mathrm{CaCl}_{2}$. Analysis was performed on fresh blood samples from donors with one concentration of dabigatran (and control samples without dabigatran) and three reversal drugs. The run time was set to 60 minutes.

\section{Measurement of clot initiation and propagation from a tissue-factor coated surface}

A method utilizing video-image capture and processing developed for the analysis of blood plasma coagulation at a tissue factor-coated surface, specially prepared with low density of tissue factor, was performed on the instrument Thrombodynamics Analyzer ${ }^{\mathrm{TM}}$ (HemaCore, Moscow, Russia). This method has been described in several articles [12-14]. Coagulation was detected in a cuvette by time-lapse image capture of light scattering from the fibrin network. By image processing and analysis, both the initiation (lag time in minutes, data not shown) and propagation phase (initial rate of clot growth; $\mu \mathrm{m} / \mathrm{min}$ ) of the coagulation process were measured in the same experiment. The experiments were done using PRP. The results were normalized due to differences between batches of reagents to enable presentation in one figure with the mean of controls for each batch of reagent set to $100 \%$.

\section{Statistics}

Results are expressed as the mean values \pm standard deviation (SD). Statistical significance was tested using the non-parametric Mann-Whitney test since the numbers may not be enough for proper testing of Gaussian distribution and is denoted as follows; $* \mathrm{P}<0.05$, ** $\mathrm{P}<0.01$, and $* * * \mathrm{P}<0.001$. 


\section{Results}

APCC shortened clotting time considerably in samples spiked with dabigatran (Fig. 1). In contrast, PCC had no effect on the prolongation caused by dabigatran (Fig. 1), but prolonged clotting time in absence of dabigatran, although not significant using Mann-Whitney test. rFVIIa shortened the clotting time in the sample spiked with dabigatran with $12 \% \pm 28 \%$ (mean change in percentage $\pm \mathrm{SD}$ ) but also shortened the clotting time in the control sample (not shown). Increasing the concentration of rVIIa three times induced further shortening of the clotting time to a reduction of $26 \% \pm 32 \%$ (Fig. 2), but the effect of tripling the concentration of rFVIIa was not significant. Dabigatran seemed to attenuate the initial rate of coagulation clot growth from 27 to $19 \mu \mathrm{m} / \mathrm{min}$ at the tissue factor-coated surface (although not significant using Mann-Whitney test). APCC increased the rate by $20 \% \pm 31 \%$ in presence of dabigatran, a significant increase using Mann-Whitney test (Fig. 3). In the presence of dabigatran, rFVIIa and PCC did not restore initial clot growth, PCC even slightly decreased the initial clot growth, although not significantly (Fig.3).

\section{Discussion}

Dabigatran is a specific inhibitor for thrombin. Agents causing large increase of the concentration of thrombin may by-pass the effect of dabigatran and may be of same use in emergency situations. We show that APCC shortened clotting time in whole blood spiked with dabigatran $(200 \mu \mathrm{g} / \mathrm{L})$, rFVIIa tended to shorten the clotting time, but not significantly, probably due to high variation and to low number of experiments. Assuming Gaussian distribution the effect of rVIIa was significant using t-test with Bonferronis correction for multiple comparisons. APCC increased the initial clot growth from a tissue-factor coated surface, although this parameter was not affected by dabigatran. In contrast, PCC had no beneficial effects in vitro on clotting time or initial clot growth vitro in samples spiked with dabigatran, but the prolongation of clotting time in absence of dabigatran was not significant. Cofact $^{\circledR}$, the PCC product used does not contain heparin in contrast to Ocplex ${ }^{\circledR}$ and Beriplex ${ }^{\circledR}$. Ocplex ${ }^{\circledR}$ prolonged clotting time much more, also in samples spiked with dabigatran. This prolongation of clotting time by Ocplex ${ }^{\circledR}$ in control samples was only partially reversed by treatment with heparinase or polybren (not shown). Thus we believe the non-significant prolongation of clotting time in absence of dabigatran is not explained by heparin in the PCCs, although we have not tried to study this further. These results are in accordance with good effects by APCC on thrombin generation parameters using the calibrated automated 
thrombography $[15,16]$ and on clotting analysis done on blood samples collected from healthy subjects infused with PCC and APCC [17]. Furthermore, the prolonged R-time in thromobelastography (which is related to clotting time) on blood samples from eight patients on dabigatran (150 mg twice daily) was shortened, although not normalized, by addition of rFVIIa but not by PCC in a recent study [18]. In addition the R-time was also prolonged in thromboelastography and ROTEM analysis in blood samples from patients on dabigatran, but effects of reversal drugs not tested in these instruments in the recent study by Herrmanns et al [16].

There is still very limited evidence for efficacy of reversal of anticoagulant effect of dabigatran. PCC improved haemostasis in a rabbit kidney injury model [19] and prevented excess intracerebral haematoma expansion in a murine intracerebral hemorrhage model [20]. PCC, APCC, rFVIIa all decreased the prolonged bleeding time in a rat tail bleeding model, but routine coagulation assays did not predict this effect. Thrombin generation reversal was dependent on the concentration of dabigatran and each reversal drug normalization occurred at the lower concentration of dabigatran with most agents, but not at higher concentrations [21]. In humans the results are less convincing. In published cases with life-threatening bleeding often more than two or more drugs potentially improving haemostasis have been used which makes it hard to evaluate the effect of each drug, as for example in reference [22], or the treatment failed as in a case with total hip replacement [23]. The clinical effect after using rFVIIa at a dose of $30 \mu \mathrm{g} / \mathrm{kg}$ in one complicated case which presented with rectal bleeding was unsatisfactory [24] as well as in one fatal case treated with dabigatran as postoperative prophylaxis [23]. In contrast, in one case of severe bleeding due to a transseptal perforation at an atrial fibrillation ablation procedure bleeding was stopped by infusion of 26 $\mathrm{U} / \mathrm{kg}$ APCC [25]. In a fatal case with dabigatran-associated intracranial hemorrhage, rFVIIa at $90 \mu \mathrm{g} / \mathrm{kg}$ body weight shortened the prolonged activated partial thromboplastin time and the prothrombin time, improved thrombin generation and shortened the R-time [26]. Case reports thus show conflicting results [23-27]. Furthermore, a very important limitation regarding case studies is that only a small fraction of cases are reported in the literature or available through other open sources.

Clinical guidelines suggest the use of PCC, APCC or rFVIIa in patients on dabigatran who suffer severe bleeding [1, 3-10]. In our opinion, based on this study and the few case reports and in vitro and ex vivo studies, for a critically bleeding patient on dabigatran our choice 
would be rFVIIa in the highest dosage used in this study to get maximal effect or APCC in same dosage as for treatment of hemophilia patients with inhibitors.

In conclusion, APCC and rFVIIa seem to have the potential for reversal of dabigatran effects on coagulation, but may in too high doses induce excessive coagulation and eventually cause thrombotic complications. PCC had less of an effect in the concentration used in this study but we cannot exclude that a higher concentration would be more efficient. There is a need for a specific reversal drug not increasing the risk for thrombosis and a monoclonal antibody has shown promising results in vitro and in animal models [2] and is in a phase III clinical study. Large registry studies and randomized clinical trials are needed to provide evidence of potential benefit and harm of the hemostatic drugs for reversal of dabigatran effects on haemostasis in patients with major or life-threatening bleeding.

\section{Acknowledgements}

The reversal drugs APCC and rFVIIa were kind gifts from the Swedish representatives of the manufacturers. The instrument Thrombodynamics Analyzer ${ }^{\mathrm{TM}}$ and reagents were provided for free by HemaCore. Cofact ${ }^{\circledR}$ and the dabigatran used in this study were kind gifts from Boehringer-Ingelheim which also provided a partial funding of the study but had no influence on the study or the article. The study was supported by grants from the County Council of Östergötland. The funding body was not involved at all in this study.

\section{References}

[1] Majeed A, Hwang HG, Connolly SJ, Eikelboom JW, Ezekowitz MD, Wallentin L, et al. Management and outcomes of major bleeding during treatment with dabigatran or warfarin. Circulation. 2013;128:2325-32.

[2] Schiele F, van Ryn J, Canada K, Newsome C, Sepulveda E, Park J, et al. A specific antidote for dabigatran: functional and structural characterization. Blood. 2013;121:3554-62.

[3] Majeed A, Schulman S. Bleeding and antidotes in new oral anticoagulants. Best Pract Res Clin Haematol. 2013;26:191-202.

[4] Steiner T, Bohm M, Dichgans M, Diener HC, Ell C, Endres M, et al. Recommendations for the emergency management of complications associated with the new direct oral anticoagulants (DOACs), apixaban, dabigatran and rivaroxaban. Clin Res Cardiol. 2013;102:399-412.

[5] Kaatz S, Kouides PA, Garcia DA, Spyropolous AC, Crowther M, Douketis JD, et al. Guidance on the emergent reversal of oral thrombin and factor Xa inhibitors. Am J Hematol. 2012;87 Suppl 1:S141-5. 
[6] Pernod G, Albaladejo P, Godier A, Samama CM, Susen S, Gruel Y, et al. Management of major bleeding complications and emergency surgery in patients on long-term treatment with direct oral anticoagulants, thrombin or factor-Xa inhibitors: proposals of the working group on perioperative haemostasis (GIHP) - March 2013. Arch Cardiovasc Dis. 2013;106:382-93.

[7] Siegal DM, Crowther MA. Acute management of bleeding in patients on novel oral anticoagulants. Eur Heart J. 2013;34:489-98b.

[8] Crowther MA, Warkentin TE. Managing bleeding in anticoagulated patients with a focus on novel therapeutic agents. J Thromb Haemost. 2009;7 Suppl 1:107-10.

[9] Fries D, Giurea A, Gutl M, Halbmayer WM, Kozek-Langenecker S, Pachucki A, et al. Management of dabigatran-induced bleeding: expert statement. Wien Klin Wochenschr. 2013;125:721-9.

[10] Schulman S, Crowther MA. How I treat with anticoagulants in 2012: new and old anticoagulants, and when and how to switch. Blood. 2012;119:3016-23.

[11] Ranby M, Ramstrom S, Svensson PO, Lindahl TL. Clotting time by free oscillation rheometry and visual inspection and a viscoelastic description of the clotting phenomenon. Scand J Clin Lab Invest. 2003;63:397-406.

[12] Soshitova NP, Karamzin SS, Balandina AN, Fadeeva OA, Kretchetova AV, Galstian GM, et al. Predicting prothrombotic tendencies in sepsis using spatial clot growth dynamics. Blood Coagul Fibrinolysis. 2012;23:498-507.

[13] Dashkevich NM, Ovanesov MV, Balandina AN, Karamzin SS, Shestakov PI, Soshitova NP, et al. Thrombin activity propagates in space during blood coagulation as an excitation wave. Biophys J. 2012;103:2233-40.

[14] Lipets E, Vlasova O, Urnova E, Margolin O, Soloveva A, Ostapushchenko O, et al. Circulating contact-pathway-activating microparticles together with factors IXa and Xla induce spontaneous clotting in plasma of hematology and cardiologic patients. PLoS One. 2014;9:e87692.

[15] Khoo TL, Weatherburn C, Kershaw G, Reddel CJ, Curnow J, Dunkley S. The use of FEIBA(R) in the correction of coagulation abnormalities induced by dabigatran. Int J Lab Hematol. 2013;35:222-4.

[16] Herrmann R, Thom J, Wood A, Phillips M, Muhammad S, Baker R. Thrombin generation using the calibrated automated thrombinoscope to assess reversibility of dabigatran and rivaroxaban. Thromb Haemost. 2014;111:989-95.

[17] Eerenberg ES, Kamphuisen PW, Sijpkens MK, Meijers JC, Buller HR, Levi M. Reversal of rivaroxaban and dabigatran by prothrombin complex concentrate: a randomized, placebo-controlled, crossover study in healthy subjects. Circulation. 2011;124:1573-9.

[18] Davis PK, Musunuru H, Walsh M, Mitra R, Ploplis V, Castellino FJ. The ex vivo reversibility of dabigatran-induced whole-blood coagulopathy as monitored by thromboelastography: mechanistic implications for clinical medicine. Thromb Haemost. 2012;108:586-8.

[19] Pragst I, Zeitler SH, Doerr B, Kaspereit FJ, Herzog E, Dickneite G, et al. Reversal of dabigatran anticoagulation by prothrombin complex concentrate (Beriplex $\mathrm{P} / \mathrm{N}$ ) in a rabbit model. J Thromb Haemost. 2012;10:1841-8.

[20] Zhou W, Schwarting S, Illanes S, Liesz A, Middelhoff M, Zorn M, et al. Hemostatic therapy in experimental intracerebral hemorrhage associated with the direct thrombin inhibitor dabigatran. Stroke. 2011;42:3594-9.

[21] van Ryn J, Schurer J, Kink-Eiband M, Clemens A. Reversal of dabigatran-induced bleeding by coagulation factor concentrates in a rat-tail bleeding model and lack of effect on assays of coagulation. Anesthesiology. 2014;120:1429-40.

[22] Mastrobuoni S, Robblee JA, Boodhwani M. Spontaneous ascending aortic intramural haematoma in a patient on dabigatran. Interact Cardiovasc Thorac Surg. 2012;15:299-300.

[23] Lillo-Le Louet A, Wolf M, Soufir L, Galbois A, Dumenil AS, Offenstadt G, et al. Life-threatening bleeding in four patients with an unusual excessive response to dabigatran: implications for emergency surgery and resuscitation. Thromb Haemost. 2012;108:583-5.

[24] Harinstein LM, Morgan JW, Russo N. Treatment of dabigatran-associated bleeding: case report and review of the literature. J Pharm Pract. 2013;26:264-9. 
[25] Dager WE, Gosselin RC, Roberts AJ. Reversing dabigatran in life-threatening bleeding occurring during cardiac ablation with factor eight inhibitor bypassing activity. Crit Care Med. 2013;41:e42-6. [26] Aron JL, Gosselin R, Moll S, Arkin CF, Mantha S. Effects of recombinant factor VIla on thrombin generation and thromboelastography in a patient with dabigatran-associated intracranial hemorrhage. J Thromb Thrombolysis. 2014;37:76-9. 


\section{FIGURES}

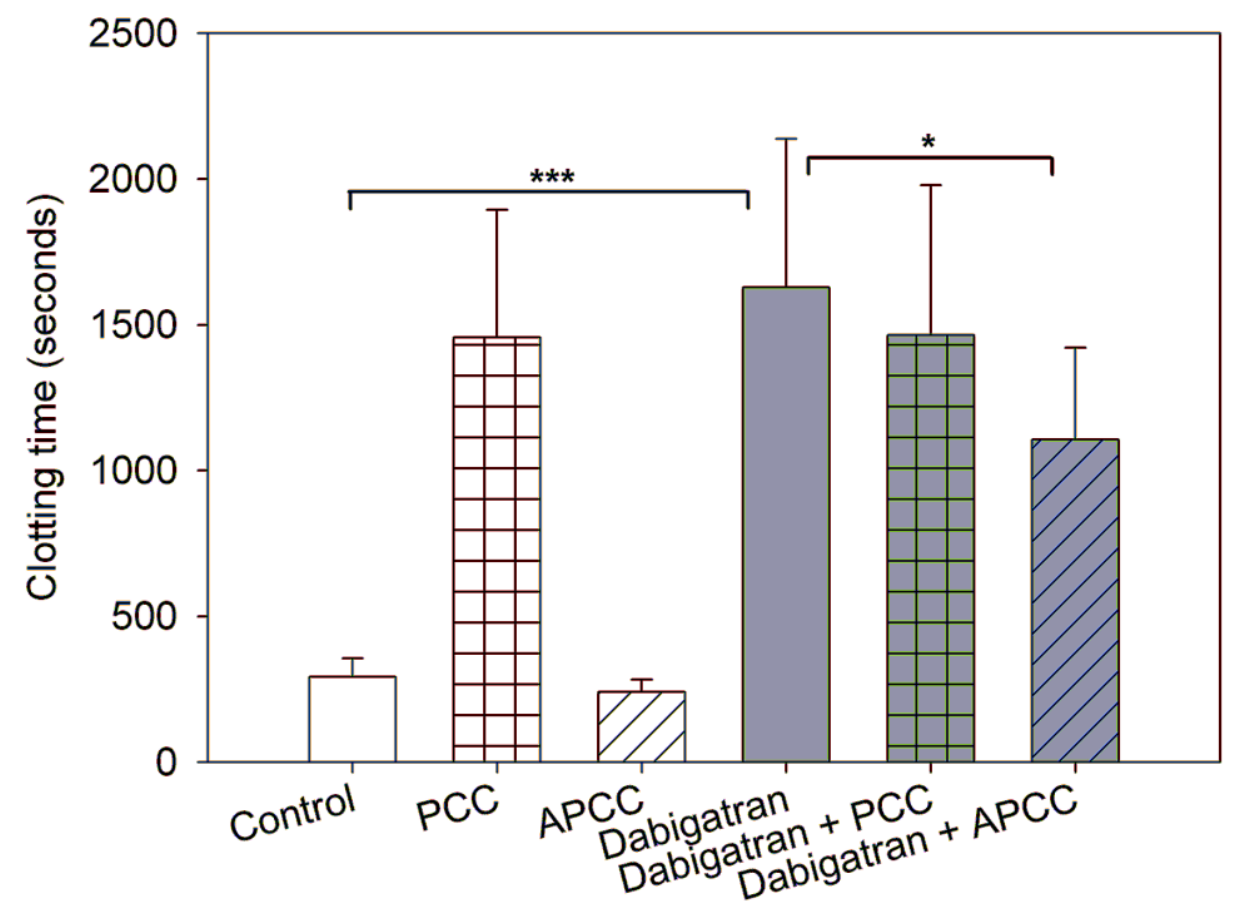

\section{Figure 1}

Activated prothrombin complex concentrate (APCC) but not prothrombin complex concentrate (PCC) shortened clotting time in blood and blood spiked with dabigatran $(200 \mu \mathrm{g} / \mathrm{L})$. Clotting was initiated with tissue factor $(1.4 \mathrm{pmol} / \mathrm{L})$, control and with dabigatran only, both $\mathrm{n}=16$. The final concentrations of reversal drugs and $\mathrm{n}$ were APCC $(1.8 \mathrm{U} / \mathrm{mL}$, $\mathrm{n}=10)$ and PCC $(0.56 \mathrm{U} / \mathrm{mL}, \mathrm{n}=16)$, respectively. Using Mann-Whitney test $* \mathrm{p}<0.05, * * \mathrm{p}<$ 0.01 and $* * * \mathrm{p}<0.001$. 


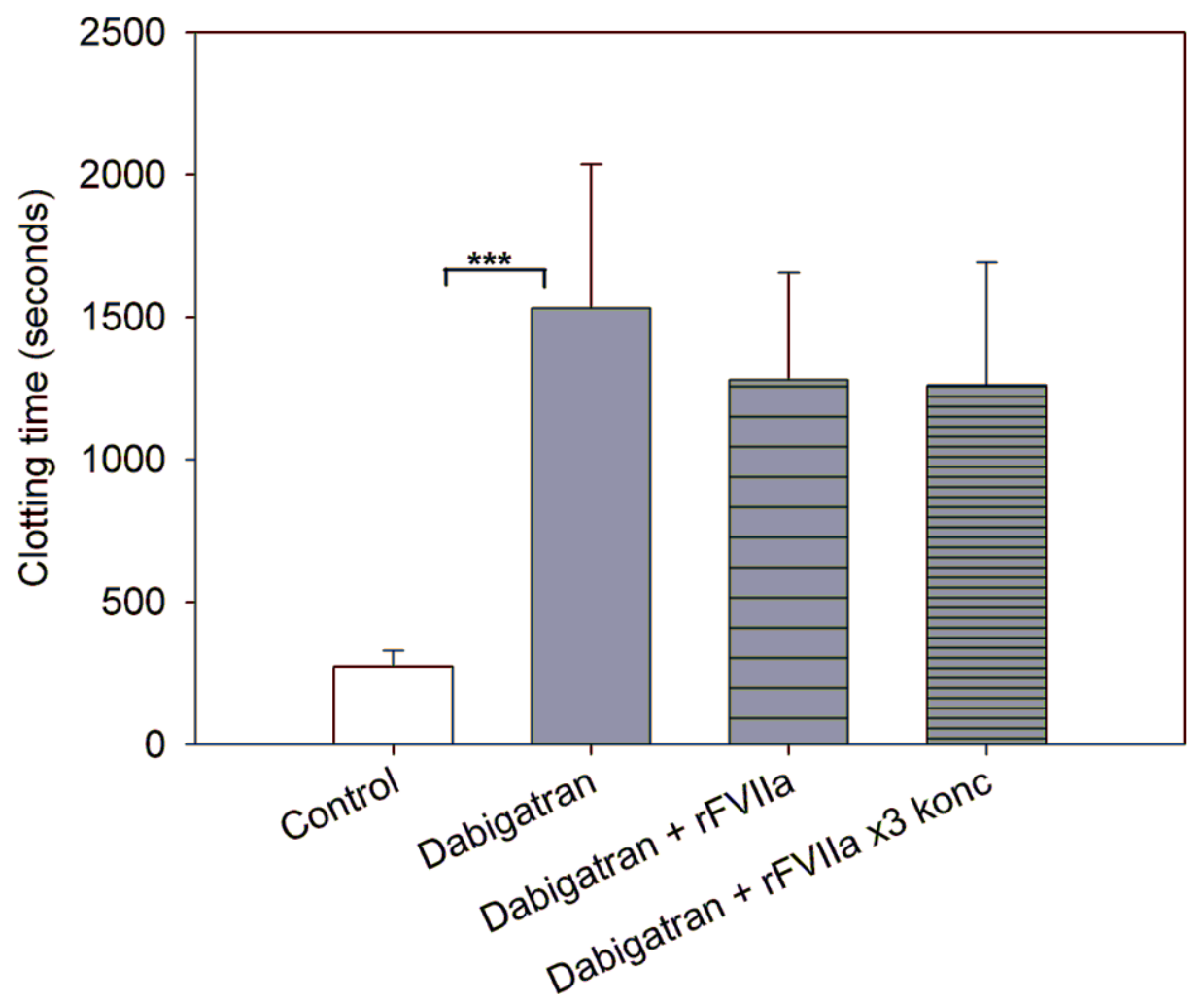

Figure 2

Recombinant factor VIIa (rFVIIa) shortened clotting times in blood and in blood spiked with dabigatran $(200 \mu \mathrm{g} / \mathrm{L})$. Clotting was initiated with tissue factor $(1.4 \mathrm{pmol} / \mathrm{L})$, control and with dabigatran only, both $n=16$. The final concentrations of the reversal drug and $n$ were rFVIIa $(2.1 \mu \mathrm{g} / \mathrm{mL}, \mathrm{n}=20$ and $6.3 \mu \mathrm{g} / \mathrm{mL}, \mathrm{n}=4)$. Using Mann-Whitney test $* * * \mathrm{p}<0.001$. 


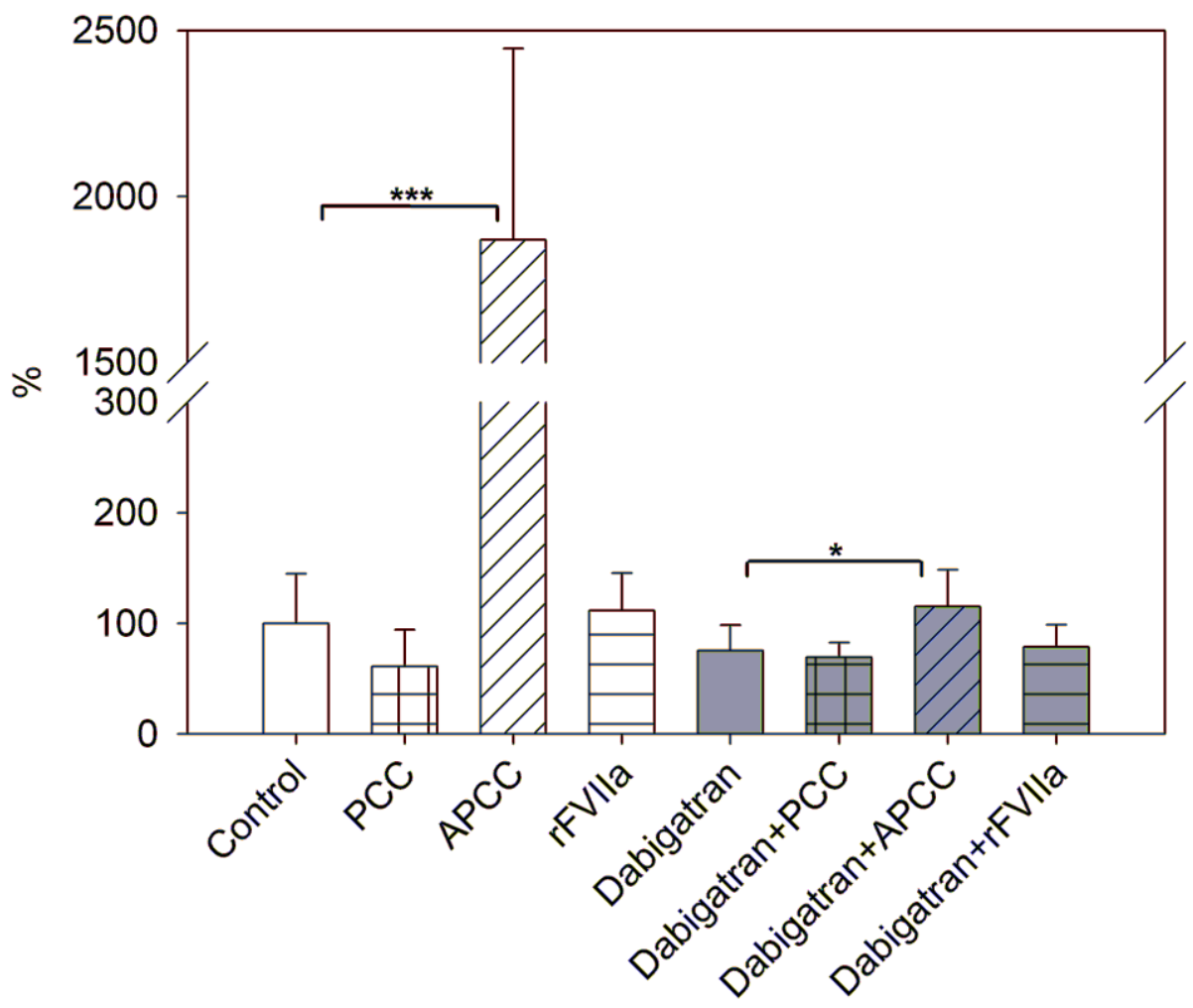

\section{Figure 3}

Initial clot growth normalized for each batch reagents with mean of controls set to $100 \%$, $n=16$. Dabigatran $(n=16)$ attenuated initial clot growth which was slightly decreased by prothrombin complex concentrate (PCC). Activated prothrombin complex concentrate (APCC) even increased initial rate of clot growth in blood with dabigatran $(200 \mu \mathrm{g} / \mathrm{L}$ significantly using Mann-Whitney test. Recombinant factor VIIa had no significant effect on initial clot growth in the presence of dabigatran $(200 \mu \mathrm{g} / \mathrm{L})$. The final concentrations of reversal drugs and $\mathrm{n}$ were APCC $(1.8 \mathrm{U} / \mathrm{mL}, \mathrm{n}=6)$ PCC $(0.56 \mathrm{U} / \mathrm{mL}, \mathrm{n}=6)$ and rFVIIa $(2.94$ $\mu \mathrm{g} / \mathrm{mL}, \mathrm{n}=12)$ Using Mann Whitney-test $* \mathrm{p}<0.05$, ** $\mathrm{p}<0.01$ and $* * * \mathrm{p}<0.001$. The small difference between control and dabigatran was not significant. 